\title{
Study on the Stair-Like Predictive Control of Train
}

\author{
Jiaxi Yuan ${ }^{1}$, Xiangxian Chen ${ }^{1}$, Chenge Geng ${ }^{1}$, Zhujun Ling ${ }^{2}$, Dandan Liu ${ }^{2}$ and Guantao $\mathrm{Hu}^{2}$ \\ ${ }^{1}$ Department of Instrumentation Science and Engineering, Zhejiang University, Hangzhou, China \\ ${ }^{2}$ Research and Development Center, Zhejiang United Science and Technology Co., Ltd, Hangzhou, China
}

\begin{abstract}
The Automatic Train Operation System is an important part of ensuring the quality of rail transit. Generalized predictive control is an algorithm which is easy to be implemented. But the classical GPC algorithm requires matrix inversion and large calculation. Based on the classical GPC, stair-like control scheme is adopted to avoid matrix inversion in this paper. Besides, input constraints are considered according to the actual situation of train operation. Therefore, the GPC algorithm can be better applied to the ATO system. The validity of the algorithm is proved by simulation.
\end{abstract}

Keywords: ATO, generalized predictive control, stair-like control, input constraints

\section{Introduction}

Rail transit is one of the most efficient means of passenger transport, which has been widely used in cities. The ATO (Automatic Train Operation) system can carry the task of operating the train automatically instead of experienced drivers. And the reliable and efficient operation of train can be achieved. The study of an effective train speed control algorithm is the basis for the realization of the ATO system with good performance.

At present, ATO control algorithm can be divided into classical control method, adaptive control method, intelligent control algorithm and so on [1]. Actually, the PID control algorithm is used in most of the ATO systems. The principles of PID are simple. Besides, it is stable and easy to implement. But the tracking/braking force has to be adjusted constantly during the operation so that it may not achieve the desired control effect [2]. At the same time, intelligent control algorithms, such as fuzzy control algorithm, often rely on the experience of experts to determine the corresponding language variables and functions. It will cause some limitations in the practical application of train control.

Therefore, in order to better apply the train control algorithm in the actual industrial production, based on the GPC (Generalized Predictive Control) algorithm, stair-like predictive control is studied in this paper. Input constraints are considered as well. Thus, a stair-like GPC algorithm with input constraints is presented and applied to train speed control.

\section{Train Prediction Model}

In general, the radius of the train track is far greater than the length of the train, and the slope of the track is small. At the same time, for the rail transit, the train usually uses the hybrid braking mode which has a good brake consistency [3]. Therefore, the train is regarded as a particle in this paper. And the prediction model of the train can be established according to Newton's law of mechanics:

\footnotetext{
${ }^{1}$ Corresponding author. Tel.: + 8615957187314.

E-mail address: sherryueda@126.com.
} 


$$
\left\{\begin{array}{l}
d v / d t=c F \\
F=u-w \\
w=\alpha_{0}+\alpha_{1} v+\alpha_{2} v^{2}
\end{array}\right.
$$

where, $v$ is the train speed, $t$ is time, $F$ is the resultant force of train, $u$ is the traction/braking force, $w$ is the resistance, $\alpha_{0}, \alpha_{1}, \alpha_{2}$ are the coefficient. follows:

Based on real-time modeling strategy, the train model represented by formal (1) can be discrete as

$$
\begin{gathered}
A\left(z^{-1}\right) y(k)=B\left(z^{-1}\right) u(k-1)+d_{0}(k)+\delta(k) \\
B\left(z^{-1}\right)=b_{1}(k) z^{-1} \\
A\left(z^{-1}\right)=1+a_{1}(k) z^{-1}
\end{gathered}
$$

where, $y(k)$ is the measured speed, $u(k)$ is the traction/braking force, $d_{0}$ is the speed deviation parameter, $\delta(k)$ is the measurement noise, $a_{1}(k) 、 b_{1}(k)$ are the parameters of the model.

\section{Stair-like GPC with input constraint}

GPC (Generalized Predictive Control) is a kind of predictive control algorithm, which is developed with the study of adaptive control. It has the characteristics of predictive model, rolling optimization and feedback correction. And it has been widely used in industrial control [4].

The classical GPC needs matrix inversion operation and has a large amount of computation, which is not suitable for the real-time system [5]. Based on the classical GPC, stair-like control scheme is adopted to avoid matrix inversion in this paper. Besides, input constraints are considered according to the actual situation of train operation. Therefore, the GPC algorithm can be better applied to the ATO system.

\subsection{Fundamentals of GPC}

According to reference [6] and reference [7], the fundamentals of GPC can be described as follows.

CARIMA model is used to predict the controlled object:

$$
A\left(z^{-1}\right) y(k)=B\left(z^{-1}\right) u(k-1)+C\left(z^{-1}\right) \delta(k) / \Delta
$$

where, $A\left(z^{-1}\right), B\left(z^{-1}\right)$ and $C\left(z^{-1}\right)$ are polynomial of operator $z^{-1}, u(k)$ and $y(k)$ are the input and output, $\Delta=1-\mathrm{z}^{-1}$ is the difference operator, $\delta(k)$ is the noise sequence.

The following Diophantine equations are introduced to get the prediction of the forward $j$ steps of the system:

$$
\begin{array}{r}
1=E_{j}\left(z^{-1}\right) A\left(z^{-1}\right) \Delta+z^{-j} F_{j}\left(z^{-1}\right) \\
E_{j}\left(z^{-1}\right) B\left(z^{-1}\right)=G_{j}\left(z^{-j}\right)+z^{-j} H_{j}\left(z^{-1}\right)
\end{array}
$$

where, $E_{j}, F_{j}, G_{j}$ and $H_{j}$ are polynomial determined by model parameters and $j$.

Through the analysis and solution of the Diophantine equations, we can get the predicted output formula as follows:

$$
y(k+j)=G_{j} \Delta u(k+j-1)+F_{j} y(k)+H_{j} \Delta u(k-1)+E_{j} \varepsilon(k+j)
$$

It can be expressed in vector form:

$$
\mathbf{Y}=\mathbf{G} \mathbf{U}+\mathbf{F} y(k)+\mathbf{H} \Delta u(k-1)+\mathbf{E}
$$

GPC is based on minimum variance optimal control, and its objective function is as follows:

$$
J=\mathrm{E}\left\{\left[\left(\mathbf{Y}-\mathbf{Y}_{r}\right)^{\mathrm{T}}\left(\mathbf{Y}-\mathbf{Y}_{r}\right)\right]+\lambda \mathbf{U}^{\mathrm{T}} \mathbf{U}\right\}=\mathrm{E}\left\{\sum_{\mathrm{j}=N_{1}}^{P}\left[y(k+j)-y_{r}(k+j)\right]^{2}+\sum_{\mathrm{j}=N_{1}}^{M} \lambda(j)[\Delta u(k+j-1)]^{2}\right\}
$$

where, $P$ is the predictive length, $M$ is the control length, and $y_{r}$ is the target value to be approached.

Minimize the objective function, and the optimal control rate is:

$$
\mathbf{U}=\left(\lambda \mathbf{I}+\mathbf{G}^{\mathrm{T}} \mathbf{G}\right)^{-1} \mathbf{G}^{\mathrm{T}}\left[\mathbf{Y}_{r}-\mathbf{F} y(k)-\mathbf{H} \Delta u(k-1)\right]
$$


Define the first line of $\left(\lambda \mathbf{I}+\mathbf{G}^{\mathrm{T}} \mathbf{G}\right)^{-1} \mathbf{G}^{\mathrm{T}}$ as:

$$
p^{\mathrm{T}}=\left[p_{1}, p_{2}, \ldots, p_{P}\right]
$$

Then, the real-time optimal control is:

$$
\begin{gathered}
u(k)=u(k-1)+\Delta u(k) \\
\Delta u(k)=p^{\mathrm{T}}\left[\mathbf{Y}_{r}-\mathbf{F} y(k)-\mathbf{H} \Delta u(k-1)\right]
\end{gathered}
$$

\subsection{Stair-like control}

In order to realize the fast calculation of GPC, the stair-like control scheme is used in this paper.

The diagram below shows the input increment in stair-like control.



Fig 1: Diagram of the input increment of stair-like control

The stair-like control scheme is introduced and the control matrix is defined as:

$$
\mathbf{Q}^{\mathrm{T}}=\left[1,1+\beta, \ldots, 1+\sum_{i=1}^{M-1} \beta^{i}\right]
$$

where, $\beta$ is the step factor. According to GPC, we can get:

$$
\begin{gathered}
\Delta u(k+j)=1+\sum_{i=1}^{j} \beta^{i} \Delta u(k) \\
\mathbf{U}^{\mathrm{T}}=\mathbf{Q}^{\mathrm{T}} \Delta u(k)=\left[1,1+\beta, \ldots, 1+\sum_{i=1}^{M-1} \beta^{i}\right] \times \Delta u(k)
\end{gathered}
$$

Let $\partial \mathbf{J} / \partial \mathbf{U}=0, \mathbf{G Q}=\mathbf{L}$, the following formula can be get:

$$
\begin{gathered}
\Delta u(k)=\left(\lambda \mathbf{Q}^{\mathrm{T}} \mathbf{Q}+\mathbf{L}^{\mathrm{T}} \mathbf{L}\right)^{-1} \mathbf{L}^{\mathrm{T}}\left[\mathbf{Y}_{r}-\mathbf{F} y(k)-\mathbf{H} \Delta u(k-1)\right] \\
u(k)=u(k-1)+\Delta u(k)
\end{gathered}
$$

Thanks to the introduction of the stair-like control matrix, the control input will change in one direction in a certain period of time, which greatly reduces the cost of the change of input and saves energy. Meanwhile, the matrix inversion is converted to scalar operation after the introduction of the stair-like control, and the actual computation is greatly reduced.

\subsection{Input constraint}

In the actual running process, the control input cannot exceed the actual maximum traction braking output. And in order to ensure the stability of the train running process, the changing degree of the traction braking of the train is limited as well.

Assuming that the input constraints are as follows:

$$
\begin{gathered}
u_{\min } \leq u(k+j) \leq u_{\max } \\
\Delta u_{\min } \leq \Delta u(k+j) \leq \Delta u_{\max }
\end{gathered}
$$

From formula (16) and formula (20), we can get:

$$
u_{\min p} \leq \Delta u(k) \leq u_{\operatorname{maxp}}
$$

where, 


$$
\begin{aligned}
& u_{\text {minp }}=\left(u_{\text {min }}-u(k-1)\right) /\left(1+\sum_{i=1}^{P-d-1} \beta^{i}\right) \\
& u_{\text {maxp }}=\left(u_{\text {max }}-u(k-1)\right) /\left(1+\sum_{i=1}^{P-d-1} \beta^{i}\right)
\end{aligned}
$$

Similarly, the formula (16) and the formula (21) can be expressed as

$$
\Delta u_{\text {min }} \leq \Delta u(k) \leq \Delta u_{\operatorname{maxp}}
$$

where

$$
\begin{gathered}
\Delta u_{\text {minp }}=\Delta u_{\text {min }} /\left(1+\sum_{i=1}^{P-d-1} \beta^{i}\right) \\
\Delta u_{\text {maxp }}=\Delta u_{\text {max }} /\left(1+\sum_{i=1}^{P-d-1} \beta^{i}\right)
\end{gathered}
$$

If the calculated $\Delta u(k)$ is out of range, then choose the boundary value.

\section{Simulation study}

According to the train control algorithm in this paper, the simulation is carried out on the train object which is based on Hangzhou Line 4. The GPC based stair-like predictive control algorithm is applied to control the train, and the effectiveness of the proposed algorithm is verified by simulation results.

Set the simulation parameters $\mathrm{P}=20, \mathrm{M}=10$. In the case of no input constraints, the simulation results of the GPC based stair-like predictive control method are shown in the following figure.



Fig. 2: Control result under the advanced GPC

As is shown in Fig. 2, the controlled speed is very close to the target speed. The stair-like GPC algorithm can track the target speed smoothly and accurately.

When there are input constraints, the stair-like GPC is applied to the same train model. Fig 3 shows the control result.



Fig. 3: Control results under stair-like GPC with input constraints

From Fig. 3, we can see that the controlled speed is quite close to the target speed even if the train is subjected to the running conditions.

The classical GPC algorithm is applied to control the train under the same condition. Comparing the change of the input and input increment of the two control method, the results are shown in Fig. 4.

In the presence of input constraints, the train can still track the target accurately and steadily under the control of the stair-like GPC algorithm. The input constraints imposed by the simulation have an impact on the stair-like GPC algorithm, which can keep the control input and input increment within the constraints. At 
the same time, the stair-like GPC with input constraints can avoid the frequent operation of the train actuator, especially in the start-up phase of the train.

According to the simulation results, we can verify that the stair-like GPC in this paper can make the results more in line with the actual situation of the train operation, and can also improve the stability of the train.
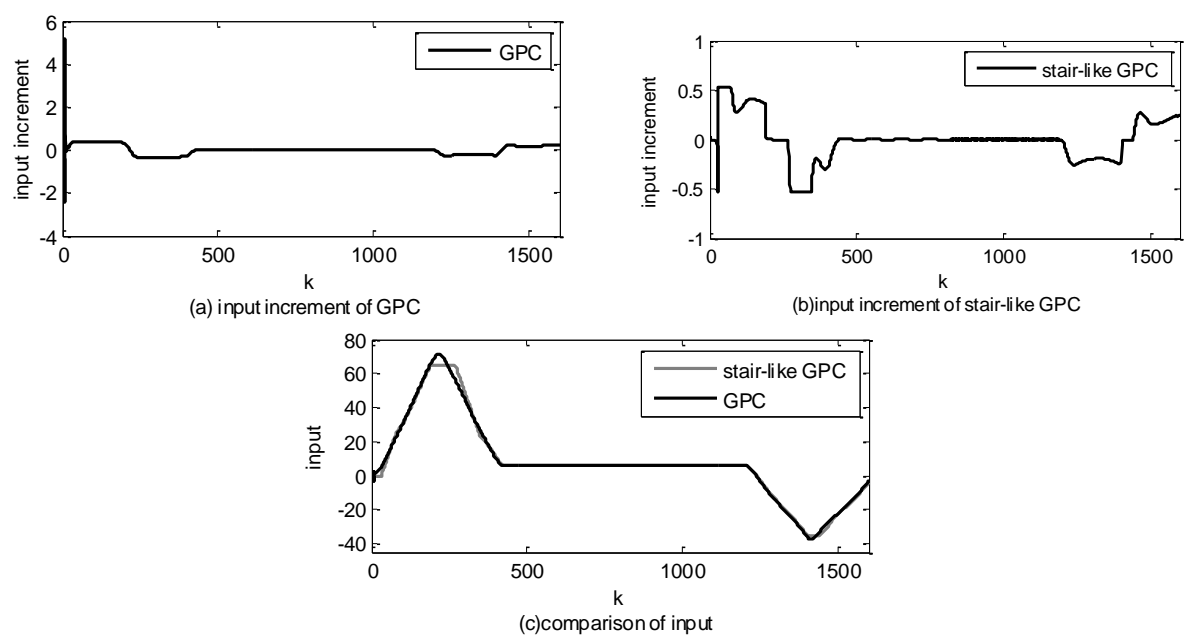

Fig. 4: Input Constraint Results

\section{Conclusion}

Aiming at the problems of input constraints in the train operation process, stair-like GPC with input constraint is applied to ATO system in this paper. The method solves the problem that the classical GPC needs to carry out a large number of matrix inverse operations. Moreover, it does a further planning of input. The simulation results show that the stair-like GPC algorithm with input constraints has a good tracking ability for a given speed target. The input can be restrained, and the input increment can be kept within the constraint condition. So that the results more in line with the actual situation of the train operation. To a certain extent, the improved GPC can improve the stability of the train operation and can meet the demand of automatic operation.

\section{Acknowledgements}

The authors wish to acknowledge Jing Zheng of Zhejiang University, who provided plenty of suggestions for this paper. This work is supported by the funding from the National Science and Technology Infrastructure Program of China under Grant 2015BAG19B03.

\section{References}

[1] T. Tao and L.J. Huang, "A Survey of Control Algorithm for Automatic Train Operation”, Journal of the China Railway Society, 2003, vol.25, pp.98-102.

[2] R. W. Chen, L. Liu and C. L. Zhu, "Automatic Train Operation and Its Control Algorithm Based on CBTC", Computer Applications, 2007, vol.27, pp.2649-2651.

[3] Z. Y. Yu and D. W. Chen, "Modeling and System Identification of Braking System of Urban Rail Vehicles", Journal of the China Railway Society, 2011, vol. 33, pp.37-40.

[4] W. Wang and J. J. Yang, "Generalized Predictive Control: Theory, Algorithm and Application", Control Theory and Application, 1997, vol. 14, pp.777-786.

[5] Q. A. Li, "Study on Simplified Implementation of Generalized Predictive Control”, Ph.D. Thesis, Zhejiang University, 2005.

[6] D. W. Clarke and C. Mohtadi, "Properties of Generalized Predictive Control", Automatica, 1989, 25(6): 859-875.

[7] D. W. Clarke, "Application of Generalized Predictive Control to Industrial Processes", IEEE Control Syst. Maga, 1988, vol. 8: 49-55. 